Pacific Journal of Mathematics

SIMPLE PERIODIC RINGS

William JaMes RAe MitcheL 


\section{SIMPLE PERIODIC RINGS}

\section{William Mitchell}

Let $A$ be a power-associative ring, and suppose that for each $a \in A$ there exists an integer $n=n(a)>1$ such that $a^{n}=a$. Such a ring $A$ is called a periodic ring. In this paper the structure of all simple periodic rings of characteristic not 2 or 3 is determined. This solves a problem posed by Osborn [Varieties of Algebras, Advances in Mathematics, to appear]. It follows from these results and from Osborn's that every flexible periodic ring with no elements of additive order 2 or 3 is a Jordan ring.

Let $A$ denote a simple periodic ring. It is shown in [1] that every element of a periodic ring has finite additive order, and it is known that any simple ring has a well-defined characteristic. Thus $A$ must be an algebra over $Z_{p}$, the integers modulo $p$, for some prime $p$. We suppose that $p \neq 2$ or 3 in order to use the results of [1]. Definitions not given here may be found in [1].

Let the multiplication give in $A$ be denoted by juxtaposition and let the operation " $o$ " in $A$ be defined by $a o b=1 / 2(a b+b a)$ for $a, b \in$ $A$. The algebra formed by taking the elements of $A$ under the same operation of addition but under the new multiplication " 0 " is denoted by $A^{+}$. It is shown in [1] that $A^{+}$is a simple Jordan algebra and that if $A$ is not a field than $A^{+}$is a periodic Jordan algebra of capacity 2. By a Jordan algebra $J$ of capacity 2 we mean a simple Jordan algebra in which there exist two orthogonal idempotents $e_{1}, e_{2}$ adding to the unity quantity and having the property that the Peirce subspaces $J_{1}\left(e_{1}\right)$ add $J_{1}\left(e_{2}\right)$ are Jordan division algebras. Periodic Jordan algebras of capacity 2 are characterized in [1] by

Proposition. Let $\Phi$ be a periodic field of characteristic not 2, let $\mu$ be a nonsquare in $\Phi$ and let $\Phi_{2}$ denote the ring of $2 \times 2$ matrices over $\Phi$. Then the Jordan subalgebra of $\Phi^{+}$consisting of the set

$$
J=\left\{\left(\begin{array}{cc}
\alpha & \beta \mu \\
\beta & \gamma
\end{array}\right) \mid \alpha, \beta, \gamma \in \Phi\right\}
$$

is a simple periodic Jordan algebra of capacity 2 over $\Phi$. Conversely, every simple periodic Jordan ring of capacity 2 and characteristic not 2 is isomorphic to $J$ for some choice of $\Phi$.

In view of this proposition we may identify the elements of $A$ with $2 \times 2$ matrices of the form 


$$
\left(\begin{array}{cc}
\alpha & \beta \mu \\
\beta & \gamma
\end{array}\right)
$$

over some periodic field $\Phi$ where $\mu$ is a fixed nonsquare of $\Phi$. Let juxtaposition of two such matrices denote the product regarding them as elements of $A$, let " 0 " denote the product regarding them as in $A^{+}$, and let "." denote the usual matrix product. Then by the above proposition these products are connected by

$$
a b+b a=2(a o b)=a \cdot b+b \cdot a
$$

for all $a, b \in A$. If $e=\left(\begin{array}{ll}1 & 0 \\ 0 & 1\end{array}\right), f=\left(\begin{array}{rr}1 & 0 \\ 0 & -1\end{array}\right), g=\left(\begin{array}{ll}0 & \mu \\ 1 & 0\end{array}\right)$, then the set $\{e, f, g\}$ will be a vector-space basis for $A^{+}$over $\Phi$. Since elements of $\Phi$ do not act as scalars on the algebra $A$, we cannot make $A$ an algebra over $\Phi$, but have to work with it as an algebra over $Z_{p}$. If $\left\{\omega_{1}, \omega_{2}, \cdots\right\}$ is a basis of $\Phi$ over $Z_{p}$, then $\left\{\omega_{1} e, \omega_{2} e, \cdots, \omega_{1} f, \omega_{2} f\right.$, $\left.\cdots, \omega_{1} g, \omega_{2} g, \cdots\right\}$ form a basis of $A$ over $Z_{p}$, and we must determine how these different basis elements multiply together.

Since we know the multiplication in $A^{+}$, we also know the product of any two elements of $A$ that commute. Most of our calculations will be to show that appropriate pairs of basis elements of $A$ commute. As any two nonzero elements of $\Phi$ may be expressed as powers of a single element of $\Phi$, it will suffice to determine the products $\lambda^{i} e \lambda^{j} e, \lambda^{i} e \lambda^{j} f, \lambda^{i} e \lambda^{j} g, \lambda^{i} f \lambda^{j} f, \lambda^{i} f \lambda^{j} g$ and $\lambda^{i} g \lambda^{j} g$ for arbitrary $\lambda \in \Phi$.

The element $e$ is the identity for $A^{+}$and the power-associativity of $A$ implies that $e$ is the identity for $A$. Hence $e e=e, e f=f=f e$, and $e g=g=g e$. Using (1) we also have

$$
f f=f \circ f=f \cdot f=f \text { and } g g=g \circ g=g \cdot g=\mu e .
$$

Since $A$ is power-associative, it satisfies the identity $x^{2} x=x x^{2}$. Defining the notation $(x, y, z) \equiv(x y) z-x(y z)$ and $[x, y]=x y-y x$, this identity can be written either as $(x, x, x)=0$ or $\left[x^{2}, x\right]=0$. The latter form has the linearization

$$
Q(x, y, z) \equiv[x \circ y, z]+[y o z, x]+[z o x, y]=0
$$

which will be of fundamental importance in subsequent calculations. We note at this point that (2) is symmetric in all variables.

We first compute the value of $\lambda^{i} e \lambda^{j} e$. Substituting $x=\lambda^{i-1} e, y=$ $\lambda e, z=\lambda^{j} e$ in (2) and using the fact that $\lambda^{r} e o \lambda^{s} e=\lambda^{r+s} e$ for $r, s$ positive integers, we obtain

$$
\left[\lambda^{i} e, \lambda^{j} e\right]+\left[\lambda^{j+1} e, \lambda^{i-1} e\right]+\left[\lambda^{i+j-1} e, \lambda e\right]=0 .
$$

This equation is generalized in 
LEMmA 1. $\left[\lambda^{i} e, \lambda^{j} e\right]+\left[\lambda^{j+l} e, \lambda^{i-l} e\right]+l\left[\lambda^{i+j-1} e, \lambda e\right]=0$ where $i, j, l$ are positive integers.

Proof. The proof is by induction on $l$. The case $l=1$ is (3). Now assume

$$
\left[\lambda^{i} e, \lambda^{j} e\right]+\left[\lambda^{j+l} e, \lambda^{i-l} e\right]+l\left[\lambda^{i+j-1} e, \lambda e\right]=0
$$

holds. Substituting $x=\lambda^{i-l-1} e, y=\lambda e, z=\lambda^{i+l} e$ in (2) gives the relation

$$
\left[\lambda^{i-l} e, \lambda^{j+l} e\right]+\left[\lambda^{j+l+1} e, \lambda^{i-l-1} e\right]+\left[\lambda^{i+j-1} e, \lambda e\right]=0 .
$$

Adding (4) and (5) gives

$$
\left[\lambda^{i} e, \lambda^{j} e\right]+\left[\lambda^{j+(l+1)} e, \lambda^{i-(l+1)} e\right]+(l+1)\left[\lambda^{i+j-1} e, \lambda e\right]=0,
$$

and hence the lemma holds by induction on $l$.

The substitution $i=p, j=(k-1) p, l=p$ in $(4)$ gives $\left[\lambda^{p} e, \lambda^{(k-1) p} e\right]+$ $0+p\left[\lambda^{k p-1} e, \lambda e\right]=0$. Since $A$ has characteristic $p$ this last equation becomes

$$
\left[\lambda^{p} e, \lambda^{(k-1) p} e\right]=0 .
$$

Let $n$ be a positive integer such that $\lambda^{p^{n}}=\lambda$. Replacing $\lambda$ by $\lambda^{p^{n-1}}$ in (6) shows that

$$
\left[\lambda e, \lambda^{k-1} e\right]=0, k \text { any positive integer . }
$$

Since $i, j, l$ are arbitrary positive integers in (4), (4) and (7) combine to give $\left[\lambda^{i} e, \lambda^{j} e\right]+\left[\lambda^{j+l} e, \lambda^{i-l} e\right]+l\left[\lambda^{i+j-1} e, \lambda e\right]=\left[\lambda^{i} e, \lambda^{j} e\right]+\left[\lambda^{j+l} e, \lambda^{i-l}\right]=$ 0 , and setting $i=l$ in this last equation gives

$$
\left[\lambda^{i} e, \lambda^{j} e\right]=0, i, j \text { positive integers . }
$$

The value of $\lambda^{i} f \lambda^{j} f$ is computed next. The substitution $x=\lambda e$, $y=z=f$ in (2) gives $[\lambda e o f, f]+[f \circ f, \lambda e]+[f o \lambda e, f]=0$ and the middle commutator is $[e, \lambda e]=0$. Hence this last equation reduces to $2[\lambda e o f, f]=[\lambda f, f]=0$, and replacing $\lambda$ by $\lambda^{i}$ generalizes this to

$$
\left[\lambda^{i} f, f\right]=0, i \text { a positive integer . }
$$

The substitution $x=\lambda^{i-1} f, y=\lambda e, z=\lambda_{f}^{j}$ in (2) gives

$$
\left[\lambda^{i} f, \lambda^{j} f\right]+\left[\lambda^{j+1} f, \lambda^{i-1} f\right]+\left[\lambda^{i+j-1} e, \lambda e\right]=0
$$

and (8) shows that the last commutator of this equation is 0 . Hence this equation reduces to $\left[\lambda^{i} f, \lambda^{j} f\right]+\left[\lambda^{j+1} f, \lambda^{i-1} f\right]=0$, and setting $i=1$ and using (9) reduces this last expression to

$$
\left[\lambda f, \lambda^{j} f\right]=0, j \text { a positive integer . }
$$


The substitution $x=\lambda^{i-1} e, y=\lambda f, z=\lambda^{j} f$ in (2) gives the equation

$$
\left[\lambda^{i-1} e o \lambda f, \lambda^{j} f\right]+\left[\lambda f o \lambda^{j} f, \lambda^{i-1} e\right]+\left[\lambda^{j} f 0 \lambda^{i-1} e, \lambda f\right]=0,
$$

and evaluating the Jordan product in these commutators in terms of Jordan product of $2 \times 2$ matrices converts this last equation to

$$
\left[\lambda^{i} f, \lambda^{j} f\right]+\left[\lambda^{j+1} e, \lambda^{j-1} e\right]+\left[\lambda^{i+j-1} f, \lambda f\right]=0
$$

which by (8) and (10) reduces to the equation

$$
\left[\lambda^{i} f, \lambda^{j} f\right]=0, i, j \text { positive integers . }
$$

We note that just as in (8), equation (11) implies

$$
\begin{aligned}
\lambda^{i} f o \lambda^{j} f & =\frac{1}{2}\left(\lambda^{i} f \lambda^{j} f+\lambda^{j} f \lambda^{i} f\right)=\frac{1}{2}\left(2 \lambda^{i} f \lambda^{j} f\right)=\lambda^{i} f \lambda^{j} f \\
& =\lambda^{i+j} f .
\end{aligned}
$$

The next case to calculate is the product $\lambda^{i} g \lambda^{j} g$. The substitution $x=\lambda^{i-1} g, y=\lambda e, z=\lambda^{j} g$ in (2) gives

$$
\left[\lambda^{i} g, \lambda^{j} g\right]+\left[\lambda^{j+1} g, \lambda^{j-1} g\right]+\left[\lambda^{i+j-1}(\mu e), \lambda e\right]=0 .
$$

But $\lambda^{i+j-1} \mu$ and $\lambda$ may be expressed as powers of a single element of $\Phi$. But then (7) shows that the last commutator in the above equation vanishes and that equation thus reduces to $\left[\lambda^{i} g, \lambda^{j} g\right]+\left[\lambda^{j+1} g\right.$, $\left.\lambda^{i-1} g\right]=0$ and setting $i=1$ in this last equation yields

$$
\left[\lambda g, \lambda^{j} g\right]+\left[\lambda^{j+1} g, g\right]=0 \text {. }
$$

Since the substitution $x=\lambda e, y=z=g$ in (2) gives $2[\lambda g, g]+[\mu e$, $\lambda e]=0$, (3) shows that this reduces to $[\lambda g, g]=0$, or more generally that $\left[\lambda^{i} g, g\right]=0$ for $i$ a positive integer. Hence (12) reduces to

$$
\left[\lambda g, \lambda^{j} g\right]=0, j \text { a positive integer } .
$$

Finally, the substitution $x=\lambda^{i-1} e, y=\lambda g, z=\lambda^{j} g$ in (2) gives

$$
\left[\lambda^{i} g, \lambda^{j} g\right]+\left[\lambda^{j+1}(\mu e), \lambda^{i-1} e\right]+\left[\lambda^{i+j-1} g, \lambda g\right]=0
$$

and the second commutator of this equation vanishes by (8) and the third commutator vanishes by (13) so that the equation reduces to

$$
\left[\lambda^{i} g, \lambda^{j} g\right]=0, i, j \text { positive integers . }
$$

To determine products of the form $\lambda^{i} e \lambda^{j} g$ make the substitution $x=\lambda^{j} g, y=\lambda^{i-1} f, z=\lambda f$ in (1) and thus obtain

$$
\left[\lambda^{j} g o \lambda^{i-1} f, \lambda f\right]+\left[\lambda^{i-1} f o \lambda f, \lambda^{j} g\right]+\left[\lambda f o \lambda^{j} g, \lambda^{i-1} f\right]=0 .
$$


Since $\lambda^{r} f o \lambda^{s} g=\lambda^{t} g o \lambda^{v} f=0$ for positive integers $r, s, t, v$ the above equation reduces to

$$
\left[\lambda^{i} e, \lambda^{j} g\right]=0, i, j \text { positive integers . }
$$

To compute products of the form $\lambda^{i} e \lambda^{j} f$ substitute $x=\lambda^{j} f, y=$ $\mu^{-1}(\lambda g), z=\lambda^{i-1} g$ in (1) to obtain

$$
\left[\lambda^{i} e, \lambda^{j} f\right]=0, i, j \text { positive integers . }
$$

In remains to determine the products of the form $\lambda^{i} f \lambda^{j} g$. In order to do this the information obtained from (2) and the powerassociative identity $\left(x^{2} x\right) x=x^{2} x^{2}$ must be studied. We first prove a preliminary lemma concerning power-associative algebras in general.

Lemma 2. Let $A$ be an algebra over a field of characteristic $\neq 2$ for which $A^{+}$is power-associative and let $x^{2} x=x x^{2}$ hold in $A$. Then $A$ is power-associative.

Proof. The partial linearization $2[x o y, x]+\left(x^{2}, y\right]=0$ of $x^{2} x=$ $x x^{2}$ holds in $A$. Setting $y=x^{2}$ gives $2\left[x o x^{2}, x\right]=0$. Hence $\left(x^{2} o x\right) x=$ $x\left(x^{2} o x\right)$. Since $x^{2} o x=x o x^{2}=x^{3}$ we have $x^{3} x=x x^{3}$ so that $x^{3} x=\left(x x^{2}\right) x=$ $x\left(x x^{2}\right)=x\left(x^{2} x\right)$. But $\left(x^{2} x\right) x=1 / 4\left[\left(x^{2} x\right) x+\left(x x^{2}\right) x+x\left(x^{2} x\right)+x\left(x x^{2}\right)\right]=$ $\left(x^{2} o x\right) o x$. However $\left(x^{2} o x\right) o x=(x o x) o(x o x)$ because $A^{+}$is power-associative. Thus $\left(x^{2} x\right) x=(x \circ x) o(x \circ x)=x^{2} x^{2}=x\left(x x^{2}\right)$. Hence $x^{2} x^{2}=\left(x^{2} x\right) x=$ $x\left(x^{2} x\right)$ holds in $A$ and $A$ is power-associative.

The products $\lambda^{i} f \lambda^{j} g$ in $A$ are now investigated in

LEMMA 3. If $\lambda \in \Phi$ then

$$
\left(\lambda^{i} f\right)\left(\lambda^{j} g\right)=f\left(\lambda^{i+j} g\right) .
$$

Thus all products of the form $\lambda^{i} f \lambda^{i} g$ are determined in $A$ once the products $f \lambda^{k} g$ are determined in $A$. Conversely if $A$ is an algebra such that $A^{+}$is a periodic Jordan algebra of capacity 2 and in which (8), (11), (14), (15), (16) and (17) hold, then $A$ is power-associative.

Proof. The substitution $x=\lambda^{i} f, y=\lambda^{j} g$ in $2[x \circ y, x]+\left[x^{2}, y\right]=$ 0 yields $\lambda^{2 i} e \lambda^{j} g=\lambda^{j} g \lambda^{2 i} e$, which is just a special case of (15). The substitution $x=\lambda^{i} g, y=\lambda^{j} f$ in $2[x o y, x]+\left[x^{2}, y\right]=0$ yields a special case of (16). Hence no information about $\lambda^{i} f \lambda^{j} g$ is obtained from the partial linearization of $x x^{2}=x^{2} x$. To obtain information from (2) for $\lambda^{i} f \lambda^{j} g$ at least one of $x, y, z$ must be of the form $\lambda^{i} f$ and at least one of the remaining two variables must be of the form $\lambda^{j} g$. Since (2) is symmetric in $x, y, z$ we may assume $x=\lambda^{i} f, y=\lambda g$ in any of the substitutions into (2) which will be of interest. For these sub- 
stitutions we note at the beginning that $\lambda^{i} f o \lambda^{j} g=0$ so that $\lambda^{i} f \lambda^{j} g=$ $-\lambda^{j} g \lambda^{i} f$. We now consider the cases.

Case 1. $x=\lambda^{i} f, y=\lambda^{j} g, z=\lambda^{k} e$ in (2) gives

$$
\left[\lambda^{j+k} g, \lambda^{i} f\right]+\left[\lambda^{i+k}, \lambda^{j} g\right]=0
$$

Case 2. $x=\lambda^{i} f, y=\lambda^{j} g, z=\lambda^{k} f$ in (2) gives a special case of (15).

Case 3. $x=\lambda^{i} f, y=\lambda^{j} g, z=\lambda^{k} g$ in (2) gives a special case of (16). Hence (18) is the only new relation derived from (2). But (18) expanded out is $\lambda^{j+k} g \lambda^{i} f+\lambda^{i+k} f \lambda^{j} g=\lambda^{i} f \lambda^{j+k} g+\lambda^{j} g \lambda^{i+k} f$, which by anticommutativity of $\lambda^{r} f$ and $\lambda^{s} g$ becomes $2\left(\lambda^{j+k} g \lambda^{i} f+\lambda^{i+k} f \lambda^{j} g\right)=0$. Thus $\lambda^{i+k} f \lambda^{j} g=-\lambda^{j+k} g \lambda^{i} f=\lambda^{i} f \lambda^{j+k} g$ and setting $i=0$ yields the relation $\left.\left(\lambda^{k} f\right)\left(\lambda^{j} g\right)=f(\lambda)^{k+j} g\right)$, which is just (17).

Formula (17) shows that once products of the form $f \lambda^{i} g$ are defined all products of the form $\lambda^{r} f \lambda^{s} g, r, s$ positive integers, are determined. However the substitutions that we have just made above into (2) do not give any information about the value of the products $f \lambda^{i} g$ themselves. We have extracted all the information that can be extracted from (2) here. Put another way, equations (8), (11), (14), (15), (16), and (17) imply that (2) holds, using the linearity of (2) and the fact that any two nonzero elements of $\Phi$ are powers of a common element. By lemma 2, if (2) holds and if $A^{+}$is power-associative, then $A$ is power-asssociative. This establishes the last statement of Lemma 3.

Stating the relations that we have derived in equations (8), (11), (14), (15), (16) and (17) for our original basis $\left\{\omega_{2} e, \omega_{2} e, \cdots, \omega_{1} f, \omega_{2} f\right.$, $\left.\cdots, \omega_{1} g, \omega_{2} g, \cdots\right\}$, we obtain

$$
\begin{gathered}
{\left[\omega_{i} e, \omega_{j} e\right]=\left[\omega_{l} e, \omega_{j} f\right]=\left[\omega_{i} e, \omega_{j} g\right]=\left[\omega_{j} f, \omega_{j} f\right]} \\
=\left[\omega_{i} g, \omega_{j} g\right]=0, \\
\left(\omega_{i} f\right)\left(\omega_{j} g\right)=f\left(\omega_{i} \omega_{j} g\right)=-\left(\omega_{j} g\right)\left(\omega_{i} f\right) .
\end{gathered}
$$

Using (1) we obtain from (19) that

$$
\begin{aligned}
\omega_{i} e \omega_{j} f & =\frac{1}{2}\left(\omega_{i} e \omega_{j} f+\omega_{j} f \omega_{i} e\right)=\frac{1}{2}\left(\omega_{i} e \cdot \omega_{j} f+\omega_{j} f \cdot \omega_{i} e\right) \\
& =\frac{1}{2} \omega_{i} \omega_{j}(e \cdot f+f \cdot e)=\omega_{i} \omega_{j} f .
\end{aligned}
$$

Doing the same thing for each other pair of basis elements that commute by (19), we obtain

$$
\begin{aligned}
\omega_{i} e \omega_{j} e & =\omega_{i} \omega_{j} e, \omega_{i} e \omega_{j} f=\omega_{i} \omega_{j} f, \omega_{i} e \omega_{j} g=\omega_{i} \omega_{j} g \\
& =\omega_{i} f \omega_{j} f=\omega_{i} \omega_{j} e, \omega_{i} g \omega_{j} g=\omega_{i} \omega_{j} \mu e
\end{aligned}
$$


for all positive integers $i$ and $j$. The products of the form $f\left(\omega_{i} g\right)$ can be chosen completely arbitrarily and the other products involving $f$ and $g$ determined by (20), since we have seen that (2) is satisfied as long as (19) and (20) are satisfied. Clearly $A^{+}$is power-associative if (21) and $\omega_{i} f o \omega_{j} g=0$ hold, and the latter is implied by (20). We sum up our result as

THEOREM 1. Let $\Phi$ be a subfield of the algebraic closure of $Z_{p}$ the integers modulo $p$ for $p \neq 2$ or 3 and let $\left\{\omega_{1}, \omega_{2}, \cdots\right\}$ be a basis of $\Phi$ over $Z_{p}$. Let $e, f, g$ be three symbots and let $A$ be the vector space over $Z_{p}$ with basis consisting of the set of symbols $\left\{\omega_{1} e, \omega_{2} e, \cdots, \omega_{1} f\right.$, $\left.\omega_{2} f, \cdots, \omega_{1} g, \omega_{2} g, \cdots\right\}$. If $\beta_{1}, \beta_{2}, \cdots$ and $\gamma_{1}, \gamma_{2}, \cdots$ and $\delta_{1}, \delta_{2}, \cdots$ are any three sequence of elements of $\Phi$, then make $A$ into an algebra by defining $f\left(\omega_{i} g\right)=\beta_{i} e+\gamma_{i} f+\delta_{i} g$ and by letting (20) and (21) hold. Then $A$ is a simple periodic ring. Conversely, every simple periodic ring of characteristic not 2 or 3 either arises in this manner or is a field.

Suppose now that our simple periodic ring $A$ is flexible, that is, $(x, y, x)=0$ for all $x, y \in A$. Then we can establish

THEOREM 2. Let $A$ be a simple periodic ring of characteristic $\neq$ 2 or 3 and let $A$ satisfy the flexible law. Then $A$ is a Jordan ring.

Proof. We may clearly assume that $A$ is not a field. Let $A$ be as constructed in Theorem 1. For $\omega_{1}, \omega_{2} \in \Phi$ the substitution $\omega_{1} f$ for $x$ and $\omega_{2} g$ for $y$ in the flexible law yields

$$
\left(\omega_{1} f \omega_{2} g\right)\left(w_{1} f\right)=-\left(\omega_{1} f\right)\left(\omega_{1} f \omega_{2} g\right) .
$$

The substitution $x=y=\omega_{2} g, z=\omega_{1} f$ in the full linearization $(x, y$, $z)+(z, y, x)=0$ of the flexible law gives

$$
\begin{aligned}
0= & \left(\omega_{2}^{2} \mu e\right)\left(\omega_{1} b\right)-\left(\omega_{2} g\right)\left(\omega_{2} \omega_{1} g f\right) \\
& +\left(\omega_{1} \omega_{2} f g\right)\left(\omega_{2} g\right)-\left(\omega_{1} f\right)\left(\omega_{2}^{2} \mu e\right),
\end{aligned}
$$

or

$$
\left(\omega_{1} \omega_{2} f g\right)\left(\omega_{2} g\right)=-\left(\omega_{1} \omega_{2} f g\right)\left(\omega_{1} \omega_{2} f g\right) .
$$

Let $\omega_{1} f \omega_{2} g=\beta e+\gamma f+\delta g$ with $\beta, \gamma, \delta \in \Phi$. Equation (22) implies that $(\beta e+\gamma f+\delta g)\left(\omega_{1} f\right)=-\left(\omega_{1} f\right)(\beta e+\gamma f+\delta g)$ and using the fact that $\phi_{1} f$ and $\phi_{2} g, \phi_{1} \in \Phi$ anticommute in $A$, this last equation reduces to $2 \omega_{1} \beta f+2 \omega_{1} \lambda e=0$. Since $e$ and $f$ are linearly independent over $\Omega$ we conclude that $\omega_{1} \beta=\omega_{1} \gamma=0$, so that $\beta=\gamma=0$. Thus $\omega_{1} f \omega_{2} g=$ $\delta g$ for some $\delta \in \Omega$. Equation (23) shows that $\delta g \omega_{2} g=-\omega_{2} g \delta g$ so 
that $2 \omega_{2} \delta \mu e=0$. Thus $\delta=0$. Hence $\omega_{1} f \omega_{2} g=0$ for every $0 \neq \omega_{1}, \omega_{2} \in \Omega$. Since (19) holds in $A$ we conclude that products of pairs of basis elements of $A$ commute. Hence the multiplication in $A$ is the same as in $A^{+}$, so $A$ is Jordan. This completes the proof of the theorem.

Theorem 2 may be used to establish the result that a periodic flexible ring with no elements of additive order 2 or 3 must be a Jordan algebra (see [1]).

\section{REFERENCE}

1. J. Marshall Osborn, Varieties of algebras, Advances in Mathematics, to appear. Received August 31, 1971.

UNIVERSITY OF WISCONSIN 


\section{PACIFIC JOURNAL OF MATHEMATICS}

\section{EDITORS}

\section{H. SAMELSON}

Stanford University

Stanford, California 94305

C. R. Новву

University of Washington Seattle, Washington 98105

\section{J. DuGundJI}

Department of Mathematics University of Southern California Los Angeles, California 90007

RICHARD ARENS

University of California Los Angeles, California 90024

\section{ASSOCIATE EDITORS}
E. F. BECKENBACH
B. H. NeumanN
F. WOLF
K. YoSHIDA

\section{SUPPORTING INSTITUTIONS}

\author{
UNIVERSITY OF BRITISH COLUMBIA \\ CALIFORNIA INSTITUTE OF TECHNOLOGY \\ UNIVERSITY OF CALIFORNIA \\ MONTANA STATE UNIVERSITY \\ UNIVERSITY OF NEVADA \\ NEW MEXICO STATE UNIVERSITY \\ OREGON STATE UNIVERSITY \\ UNIVERSITY OF OREGON \\ OSAKA UNIVERSITY
}

\author{
UNIVERSITY OF SOUTHERN CALIFORNIA \\ STANFORD UNIVERSITY \\ UNIVERSITY OF TOKYO \\ UNIVERSITY OF UTAH \\ WASHINGTON STATE UNIVERSITY \\ UNIVERSITY OF WASHINGTON \\ $*{ }^{*}$
AMERICAN MATHEMATICAL SOCIETY
NAVAL WEAPONS CENTER
}

The Supporting Institutions listed above contribute to the cost of publication of this Journal, but they are not owners or publishers and have no responsibility for its content or policies.

Mathematical papers intended for publication in the Pacific Journal of Mathematics should be in typed form or offset-reproduced, (not dittoed), double spaced with large margins. Underline Greek letters in red, German in green, and script in blue. The first paragraph or two must be capable of being used separately as a synopsis of the entire paper. The editorial "we" must not be used in the synopsis, and items of the bibliography should not be cited there unless absolutely necessary, in which case they must be identified by author and Journal, rather than by item number. Manuscripts, in duplicate if possible, may be sent to any one of the four editors. Please classify according to the scheme of Math. Rev. Index to Vol, 39. All other communications to the editors should be addressed to the managing editor, Richard Arens, University of California, Los Angeles, California, 90024.

50 reprints are provided free for each article; additional copies may be obtained at cost in multiples of 50 .

The Pacific Journal of Mathematics is issued monthly as of January 1966. Regular subscription rate: $\$ 48.00$ a year (6 Vols., 12 issues). Special rate: $\$ 24.00$ a year to individual members of supporting institutions.

Subscriptions, orders for back numbers, and changes of address should be sent to Pacific Journal of Mathematics, 103 Highland Boulevard, Berkeley, California, 94708.

PUBLISHED BY PACIFIC JOURNAL OF MATHEMATICS, A NON-PROFIT CORPORATION

Printed at Kokusai Bunken Insatsusha (International Academic Printing Co., Ltd.), 270, 3-chome Totsuka-cho, Shinjuku-ku, Tokyo 160, Japan. 


\section{Pacific Journal of Mathematics}

\section{Vol. 44, No. $2 \quad$ June, 1973}

Tsuyoshi Andô, Closed range theorems for convex sets and linear liftings . . . . . . 393

Richard David Bourgin, Conically bounded sets in Banach spaces . . . . . . . . . 411

Robert Jay Buck, Hausdorff dimensions for compact sets in $R^{n} \ldots \ldots \ldots \ldots \ldots \ldots . \ldots 421$

Henry Cheng, A constructive Riemann mapping theorem ................ 435

David Fleming Dawson, Summability of subsequences and stretchings of

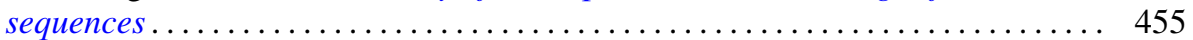

William Thomas Eaton, A two sided approximation theorem for 2-spheres ....... 461

Jay Paul Fillmore and John Herman Scheuneman, Fundamental groups of compact complete locally affine complex surfaces ....................... 487

Avner Friedman, Bounded entire solutions of elliptic equations . . . . . . . . . . . 497

Ronald Francis Gariepy, Multiplicity and the area of an $(n-1)$ continuous

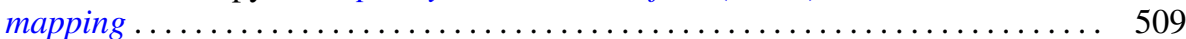

Andrew M. W. Glass, Archimedean extensions of directed interpolation groups . . . . 515

Morisuke Hasumi, Extreme points and unicity of extremum problems in $H^{1}$ on

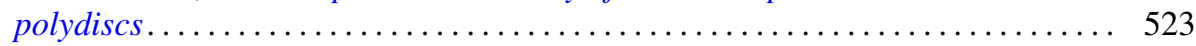

Trevor Ongley Hawkes, On the Fitting length of a soluble linear group . . . . . . 537

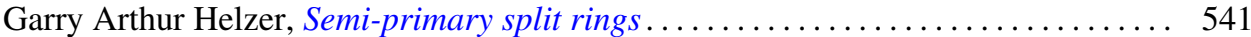

Melvin Hochster, Expanded radical ideals and semiregular ideals . . . . . . . . . 553

Keizō Kikuchi, Starlike and convex mappings in several complex variables . . . . . . 569

Charles Philip Lanski, On the relationship of a ring and the subring generated by its

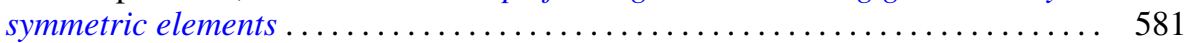

Jimmie Don Lawson, Intrinsic topologies in topological lattices and semilattices ........................................... 593

Roy Bruce Levow, Counterexamples to conjectures of Ryser and de Oliveira ...... 603

Arthur Larry Lieberman, Some representations of the automorphism group of an infinite continuous homogeneous measure algebra ..........

William George McArthur, $G_{\delta}$-diagonals and metrization theorems $\ldots .$.

James Murdoch McPherson, Wild arcs in three-space. II. An invariant of

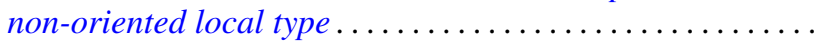

H. Millington and Maurice Sion, Inverse systems of group-valued measures ...

C. Edward Moore, Concrete semispaces and lexicographic separation of convex

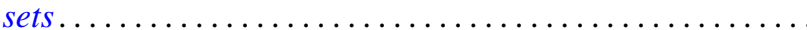

Jingyal Pak, Actions of torus $T^{n}$ on $(n+1)$-manifolds $M^{n+1}$.

Merrell Lee Patrick, Extensions of inequalities of the Laguerre and Turán type . . . . 675

Harold L. Peterson, Jr., Discontinuous characters and subgroups of finite index. . . . 683

S. P. Philipp, Abel summability of conjugate integrals . . . . . . . . . . . . . 693

R. B. Quintana and Charles R. B. Wright, On groups of exponent four satisfying an

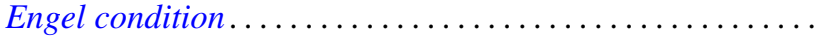

Marlon C. Rayburn, On Hausdorff compactifications. . . . . . . . . .

Martin G. Ribe, Necessary convexity conditions for the Hahn-Banach theorem in

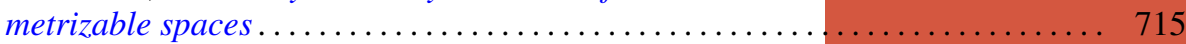

Ryōtarō Satō, On decomposition of transformations in infinite measure spaces .... 733

Peter Drummond Taylor, Subgradients of a convex function obtained from a

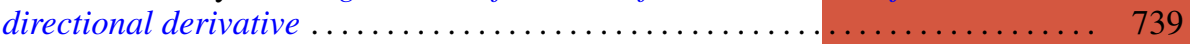

James William Thomas, A bifurcation theorem for $k$-set contractions . . . . . . . . 749 Clifford Edward Weil, A topological lemma and applications to real functions . . . . 757

Stephen Andrew Williams, A nonlinear elliptic boundary value problem . . ....... 767

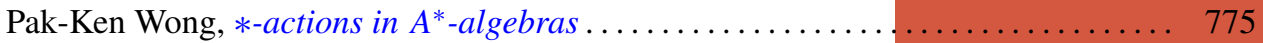

